\title{
BMJ Open Hospital at Home care for older patients with cognitive impairment: a protocol for a randomised controlled feasibility trial
}

\author{
Maaike A Pouw, ${ }^{1,2}$ Agneta H Calf, ${ }^{1}$ Barbara C van Munster, ${ }^{1,3}$ Jan C ter Maaten, ${ }^{4}$ \\ Nynke Smidt, ${ }^{1,5}$ Sophia E de Rooij ${ }^{1}$
}

To cite: Pouw MA, Calf AH, van Munster BC, et al. Hospital at Home care for older patients with cognitive impairment: a protocol for a randomised controlled feasibility trial. BMJ Open 2018;8:e020332. doi:10.1136/ bmjopen-2017-020332

- Prepublication history for this paper is available online. To view these files, please visit the journal online (http://dx.doi. org/10.1136/bmjopen-2017010332).

Received 30 0ctober 2017 Revised 8 February 2018 Accepted 12 February 2018

\section{Check for updates}

1Department of Geriatrics, University Medical Center Groningen, University of Groningen, Groningen, The Netherlands

${ }^{2}$ Department of Internal Medicine, Martini Hospital, Groningen, The Netherlands

${ }^{3}$ Department of Geriatrics, Gelre Hospitals, Apeldoorn, The Netherlands

${ }^{4}$ Department of Internal

Medicine, Emergency

Department, University of

Groningen, University Medical

Center Groningen, Groningen,

The Netherlands

${ }^{5}$ Department of Epidemiology, University of Groningen, University Medical Center Groningen, Groningen, The Netherlands

\section{Correspondence to}

Maaike A Pouw;

m.a.pouw@umcg.nl and Sophia

E de Rooij;

s.e.j.a.de.rooij@umcg.nl

\section{ABSTRACT}

Introduction An acute hospital admission is a stressful life event for older people, particularly for those with cognitive impairment. The hospitalisation is often complicated by hospital-associated geriatric syndromes, including delirium and functional loss, leading to functional decline and nursing home admission. Hospital at Home care aims to avoid hospitalisation-associated adverse outcomes in older patients with cognitive impairment by providing hospital care in the patient's own environment. Methods and analysis This randomised, non-blinded feasibility trial aims to assess the feasibility of conducting a randomised controlled trial in terms of the recruitment, use and acceptability of Hospital at Home care for older patients with cognitive impairment. The quality of care will be evaluated and the advantages and disadvantages of the Hospital at Home care programme compared with usual hospital care. Eligible patients will be randomised either to Hospital at Home care in their own environment or usual hospital care. The intervention consists of hospital level care provided at patients' homes, including visits from healthcare professionals, diagnostics (laboratory tests, blood cultures) and treatment. The control group will receive usual hospital care. Measurements will be conducted at baseline, during admission, at discharge and at 3 and 6 months after the baseline assessment.

Ethics and dissemination Institutional ethics approval has been granted. The findings will be disseminated through public lectures, professional and scientific conferences, as well as peer-reviewed journal articles. The study findings will contribute to knowledge on the implementation of Hospital at Home care for older patients with cognitive disorders. The results will be used to inform and support strategies to deliver eligible care to older patients with cognitive impairment.

Trial registration number NTR6581; Pre-results.

\section{INTRODUCTION}

\section{Background}

An acute hospital admission is a stressful life event, particularly for older people. In addition to the stress of an acute illness, the hospital admission itself contributes to this stress. ${ }^{1}$ Older hospitalised patients are often

\section{Strengths and limitations of this study}

- This study addresses the feasibility of Hospital at Home care in patients with cognitive impairment, a patient population that is often excluded from participation in scientific research.

- A process evaluation facilitates the investigation of factors that influence the experiences and perceptions of all persons involved in Hospital at Home care.

- Stakeholders were involved in the development of the design of the study which will support the implementation of Hospital at Home care and a future trial.

- Because of a limited sample size due to the study being centred on feasibility, results will not show effectiveness of Hospital at Home care compared with usual hospital care.

deprived of sleep, and they spend an average of 20 of every 24 hours in bed, they become poorly nourished, and experience sensory deprivation or overstimulation, resulting in confusion. ${ }^{2-5}$ These adverse effects of hospitalisation contribute to the occurrence of geriatric conditions, such as delirium, functional decline, falls, incontinence, hospital acquired infections and pressure injuries. ${ }^{6-9}$ Adverse effects of hospitalisation occur more easily in older people, particularly in those who are already frail, a growing portion of the worldwide ageing population. ${ }^{10}$ Frailty is a state of increased vulnerability to external stressors resulting from ageing-associated declines in reserve and function across multiple physiological systems. ${ }^{10}$ Cognitive impairment (ie, dementia) is an important contributor to frailty in older people. ${ }^{12}$ Cognitively impaired older people are more likely to become hospitalised and once admitted, they experience longer stays than their peers without cognitive impairment. ${ }^{13-15}$ The combination of hospitalisation and cognitive impairment 
in older people is associated with further functional and cognitive declines and higher mortality rates, and it leads to more discharges to long-term care facilities. ${ }^{16}{ }^{17}$ The prevalence and worldwide burden of cognitive impairment will continue to increase as the average life expectancy increases. ${ }^{18}$ The total number of people with cognitive impairment is estimated to be 75.6 million in 2030 and will nearly triple in 2050 to 135.5 million. ${ }^{19}$ An increase in the number of hospital admissions of older people with cognitive impairment and an increase in number of hospitalisation-associated adverse outcomes are therefore to be expected.

Besides adverse outcomes of hospitalisation, many older people and their caregivers do not necessarily desire a hospital admission in case of an acute illness or exacerbation of a chronic illness. Fried et al (2000) have studied the preferences of community-dwelling persons 65 years of age and older who were hospitalised with a primary diagnosis of congestive heart failure, chronic obstructive pulmonary disease (COPD) or pneumonia. The authors reported that over $50 \%$ of older patients preferred to receive hospital treatment at home, because they felt that their homes were more comfortable. ${ }^{20}$ In the treatment preferences of seriously ill patients aged 60 years and older, the likelihood of cognitive and functional impairment as an adverse outcome of the treatment was weighed in the decision-making process. There was a substantial decrease in the number of participants who opted for treatment if the likelihood of impairment after treatment was $50 \%$ or higher. ${ }^{21}$

Hospital at Home care could provide an effective alternative to inpatient care for a select group of elderly patients now requiring hospitalisation. Hospital at Home care is coordinated, multidisciplinary care in the homes of people who would otherwise be admitted to the hospital. Hospital at Home care is an accepted alternative to inpatient hospital level care in several countries (eg, the USA, Italy and the UK) but not yet in the Netherlands. ${ }^{22}$ Since the 1990s, Hospital at Home has been evaluated in (older) persons with various acute medical conditions, such as heart failure, exacerbations in COPD and infections (eg, cellulitis, pneumonia). ${ }^{22}$ In systematic reviews comparing alternative strategies to inpatient hospitalisation, lower or equal mortality rates and return hospitalisation rates (ie, subsequent admissions after discharge) were found for Hospital at Home care, there was a lower incidence of delirium, and there was a positive effect on patient and caregiver satisfaction. ${ }^{22-24}$ Only one completed trial conducted in Italy included 109 patients with cognitive impairment (ie, dementia), Tibaldi et al reported a positive effect of a Hospital at Home intervention on behavioural disturbances and caregiver stress in patients with dementia. ${ }^{25}$ Results of a still ongoing trial including people with i.a. cognitive impairment in the UK, will follow in the near future. ${ }^{26}$

Whether Hospital at Home care provides a suitable alternative with regard to other outcomes as patient satisfaction, quality of care, hospitalisation-associated adverse events and costs in older people with cognitive impairment remains unclear and further research is needed. Therefore, our primary aim is to investigate the feasibility of a Hospital at Home care programme for older patients with cognitive impairment in terms of the patient recruitment, use and acceptability, and second to investigate the advantages and disadvantages of Hospital at Home care compared with usual hospital care from different perspectives.

Objectives of this study are the following:

1. To assess the participation rate of the Hospital at Home trial among patients 65 years and older with cognitive impairment, acute illness and emergency hospital admission. What are the reasons for non-participation?

2. To assess the potential advantages and disadvantages of Hospital at Home care and usual hospital care for the patients, caregivers and Dutch medical health system.

3. To assess the feasibility of Hospital at Home care in terms of the quality of care with regard to geriatric syndromes, institutionalisation, mortality, total days with urinary catheter, length of stay (in the hospital or in Hospital at Home care) and timing/intensity of the contact with healthcare professionals.

\section{METHODS AND ANALYSIS}

\section{Trial design}

The design is a randomised controlled feasibility trial and will use a process evaluation. This study will be conducted at the medical emergency department (ED) of the academic hospital of the University Medical Center of Groningen in the Netherlands and will evaluate cognitively impaired older patients who are in need of acute hospital care. Figure 1 shows the trial design summary. Participants will be randomised to either Hospital at Home care or usual hospital care in a 4:1 ratio, respectively. Patients will be randomised using a computerised random number generator (http://www.randomization. com), including block randomisation.

An independent research nurse who is not involved in the patient care will complete the baseline assessment and allocate the participants (using sealed sequenced envelopes) into the Hospital at Home care (intervention) or usual hospital care group (control). The research nurse will not be aware of the randomisation method. The participants, healthcare professionals and research staff will not be blinded to the intervention. The reporting of the design of this trial protocol is in accordance with the SPIRIT (Standard Protocol Items: Recommendations for Interventional Trials) 2013 statement for clinical trial protocols. $^{27}$

\section{Study population}

Patients 65 years of age and older who are admitted to the medical ED will be identified by the ED staff as potential eligible patients. Subsequently, the ED staff will inform the research nurse. The research nurse will 


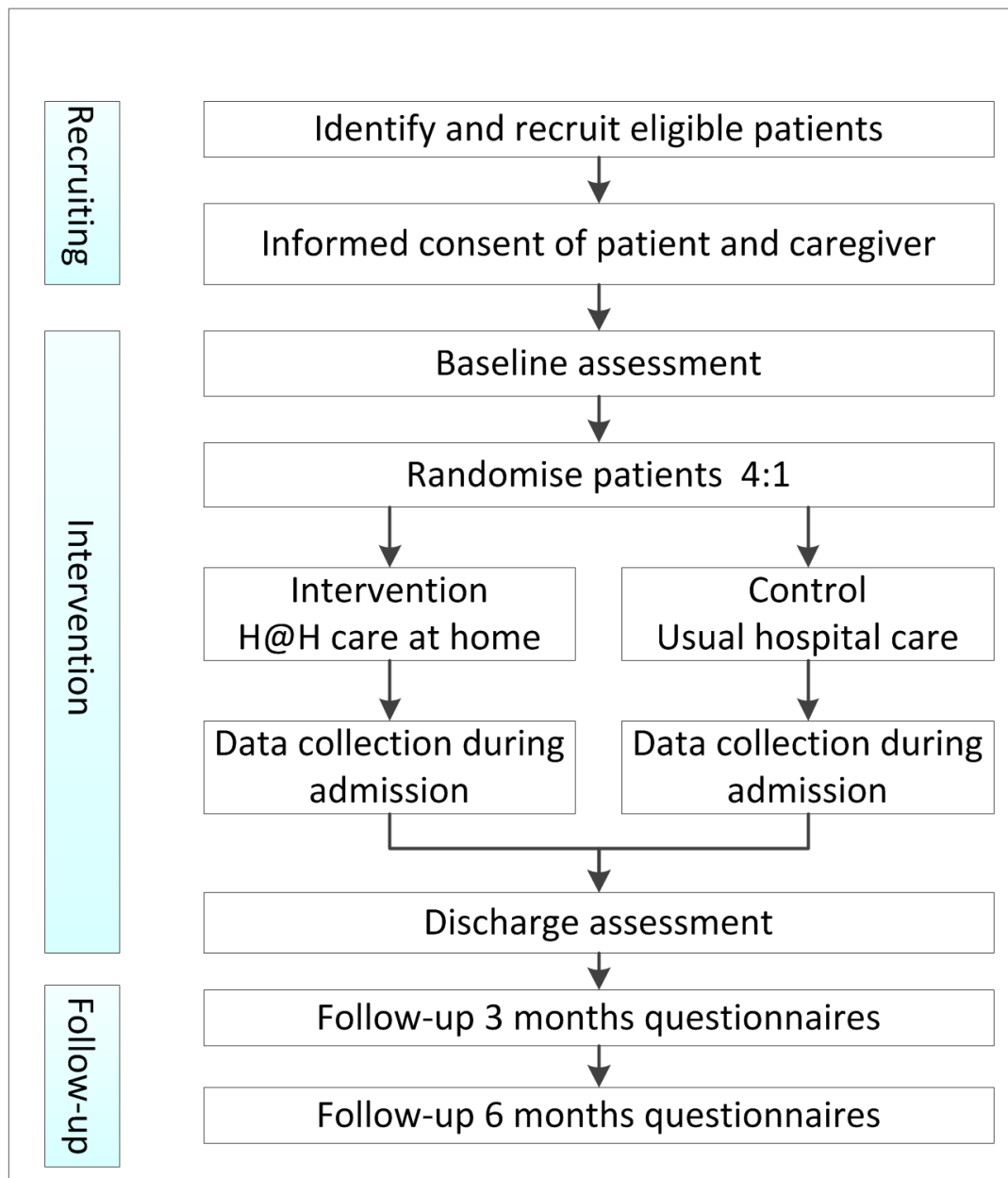

Figure 1 Flow chart of trial design summary

complete the eligibility criteria checklist (table 1 ). The research nurse will ask the patient and their informal caregiver (ie, partner, child, relative, friend) for their willingness to participate in the study and to provide written informed consent. The patient and informal caregiver will need to both sign the informed consent form before the patient can participate in the $\mathrm{H} @ \mathrm{H}$ trial.

Table 1 Patient eligibility criteria for participation in Hospital at Home trial

\begin{tabular}{|c|c|}
\hline Inclusion criteria & Exclusion criteria \\
\hline $\begin{array}{l}\text { Age } 65 \text { years of age and older } \\
\text { - Cognitive impairment, that is, dementia, delirium or other cause } \\
\text { of cognitive impairment, and either Previously diagnosed or } \\
\text { documented in the medical records or } \\
\text { - Identified by the ED clinician (eg, with the } 4 \text { AT test and/or six-item } \\
\text { cognitive impairment test) }{ }^{42-44} \\
\text { Presented at the ED with a defined acute illness } \\
\text { - Required hospital admission, according to the attending } \\
\text { ED physician but not expected to require emergency } \\
\text { interventions, Modified Early Warning Score } \leq 2 \text { points }{ }^{45-47} \\
\text { Living in hospital's catchment area (<25 km) } \\
\text { Informal caregiver is present and able to understand and perform } \\
\text { instructions and consented to participate in the trial } \\
\text { Home suitable for Hospital at Home care (available informal } \\
\text { caregiver, running water, adequate heating, safety) }{ }^{44}\end{array}$ & $\begin{array}{l}\text { Previously enrolled } \\
\text { prespitalised within the } 7 \text { days preceding ED } \\
\text { presentation } \\
\text { pursing home residents or awaiting a nursing home } \\
\text { sleeping waiting list candidates) }{ }^{27} \\
\text { - Additional care needed: Required surgical } \\
\text { assessment } \\
\text { - Suspected acute coronary syndrome or cardiac } \\
\text { arrythmia }{ }^{45} \\
\text { - Dialysis dependent patients }{ }^{45} \\
\text { - Expected terminal events } \\
\text { diagnostic or in nealliative care due to oncological or } \\
\text { haematological illness }\end{array}$ \\
\hline
\end{tabular}

ED, emergency department. 
An evaluation to assess the mental capacity is conducted by the involved ED staff in the setting of the ED assessment. If the participant lacks the capacity to consent (mentally incapacitated), and an informal caregiver (ie, partner, child, friend) is present, this informal caregiver will be asked to act as a personal consultee. The personal consultee will determine whether he or she believes that participation in the study would be in accordance with the values and interests of the individual and will subsequently sign the patient's informed consent form.

\section{Sample size of study population}

Based on the numbers available from the ED of the University Medical Center Groningen, the Netherlands, we calculated that an average of 3990 older patients 65 years and over is admitted to our medical ED each year. The Hospital at Home trial will be introduced during working hours, which provides an estimated 1900 patients per year. Not all 1900 patients will be eligible for study inclusion. Based on screening of ED medical records, approximately $15 \%$ of the patients meet the eligibility criteria for Hospital at Home care, resulting in 285 eligible persons per year. In recent randomised controlled trials (RCTs) of Hospital at Home care in Italy, $54 \%$ and $57 \%$ of the eligible patients was willing to participate and gave informed consent. ${ }^{28}{ }^{29}$ We presume a similar consent rate of $50 \%$, as described in these previous clinical trials, and expect for 143 patients to be included.

\section{Study procedures}

After (written) informed consent is obtained, all participants will complete two brief tests to assess cognitive impairment, and the participant and caregiver will complete the baseline assessment. Subsequently, randomisation takes place to either (1) the Hospital at Home care-intervention, translocation of care from the hospital to a participants' home or (2) the control group, usual hospital care. All care will be delivered according to hospital protocols, current regulations and guidelines and, if needed, described in the standard operating procedures (SOPs). If no informed consent is given by either the patient or the caregiver, the reasons for non-participation, date of birth, sex of the patient and the relationship between patient and caregiver will be reported.

\section{INTERVENTION}

\section{Hospital at Home care}

Hospital at Home care will be delivered by a multidisciplinary team consisting of a physician, nurse, pharmacist and physiotherapist. Depending on the participants' needs, other disciplines (eg, a dietician, occupational therapist or social services) can be involved in the Hospital at Home care. The day-to-day care will be provided by the nurse and physician visiting the participant. The Hospital at Home care team works under the responsibility of the medical specialist in the hospital, and $24 / 7$ consultation of the expertise and services of the hospital is part of the protocol.

The participants allocated to Hospital at Home care will receive hospital level care in their own homes. After a stay of one night in the hospital, while the Hospital at Home care arrangements are being made, the participant will be transferred home and receive Hospital at Home care. The Hospital at Home nurse is responsible for the day-to-day care and will be present on arrival of the participant at his/her residence. Hospital at Home care is described in the care protocols including SOPs and could include intravenous therapy (eg, antibiotics, fluid, and/or diuretics), oxygen therapy, and/or nebuliser, indwelling urine catheter or a nasopharyngeal food tube. After the care intake and a period of direct nursing supervision, the participant will receive intermittent nursing visits daily (starting with three times per day), including weekends and public holidays. The Hospital at Home physician will make a home visit every day (excluding weekends). The Hospital at Home physician and nurse will be available for emergency visits. The participant will receive a medical alert device in the house, with a 24/7 connection to an on-call service. Alert instructions will be explained to the participant and caregiver. A physiotherapist will visit the participant at home to evaluate any problems with balance and/or walking and immobility. The Hospital at Home team works under the supervision of the hospital medical specialist. Daily screenings and measurements will be recorded in a Hospital at Home record, which stays with the participant. Diagnostic procedures and therapeutics that cannot wait and are not available at home, such as endoscopy or CT scan, will be arranged through brief visits to the hospital. The participant will be 'admitted' to the Hospital at Home care for as long as indicated.

\section{Discharge from Hospital at Home care}

If the participant recovers to such an extent that hospital level care is no longer needed, the participant will be discharged from the Hospital at Home care programme, similar to the discharge procedure when the participant would receive usual hospital care. Hospital at Home care will end with discharge planning with the participant, family, Hospital at Home physician and nurse. The discharge plan includes follow-up appointments (eg, at the hospital or general practitioner), information on medication, warning signs and symptoms and an ongoing management plan. All hospital-related care equipment will be removed from the participants' house, and arrangements with home care agencies and/or paramedical staff will be reviewed and adjusted to the current situation.

\section{Hospital care as usual}

Participants allocated to the control arm will be admitted to a hospital ward and receive usual hospital care. After admission and intake on the ward, the participant will receive intermittent visits from the ward nurse multiple 
times a day. The ward physician will visit once every day (excluding weekends), with extra visits provided if needed. An emergency alert device, through which nurses and physician can be contacted, will be placed next to the bed. A physiotherapist will visit the participant at the ward to address problems with balance and/or walking and immobility. Depending on the participants' needs, other disciplines (eg, a dietician, occupational therapist or social services) can be involved in the hospital care. The medical record is the hospital record and additional $\mathrm{H} @ \mathrm{H}$ research forms will be added to this record for research purposes. The participant will be admitted to the hospital for as long as indicated.

\section{Hospital discharge}

If the participant recovers to such an extent that hospital level care is no longer needed, the participant will be discharged from the hospital after discharge planning with the participant, family, physician and nurse. The discharge plan includes follow-up appointments (eg, at the hospital or general practitioner), information on medication, warning signs and symptoms and an ongoing management plan. Arrangements with home care agencies and/or paramedical staff will be reviewed and adjusted to the current situation.

\section{Follow-up}

At 3 and 6 months following randomisation, all participants will be contacted for an interview by telephone ${ }^{30}$ or a face-to-face interview, if needed. The participants will be allowed to receive support with these questions from their relatives or informal caregivers. An interview will require a maximum of $30 \mathrm{~min}$. In case of institutionalisation or mortality, this event will be recorded. Additionally, information on hospital readmission and length of stay will be collected from the hospital administration system and health insurers. Mortality and nursing home placements will be collected from registries from the general practitioner and municipalities.

\section{Timing of measurements and outcome measures}

Data will be collected at baseline at the ED, during admission (in Hospital at Home or hospital), at discharge and at 3 and 6 months following randomisation, plus or minus 2 weeks. An overview of the timing of measurements and outcome measures are shown in table 2.

\section{Feasibility}

For the participation rates, the proportion of participants per step will be calculated. The reasons for non-participation and data concerning the characteristics of non-participants will be collected. We consider the participation rate feasible when it is similar to the participation rate as is described in previous RCTs and around $50 \%$ of the eligible patients will consent to participate. ${ }^{28}{ }^{29}$ Quality of care will be measured by collecting data on patient, institutionalisation (eg, to the hospital or nursing home), mortality, activities of daily living functioning, prevalence of hospitalisation-associated geriatric syndromes, the length of stay in the hospital or Hospital at Home care programme and contact with healthcare professionals. The study is considered feasible if the quality of care of Hospital at Home care on these measurements is non-inferior to usual hospital care.

\section{Other outcomes measures}

Advantages and disadvantages of the Hospital at Home care programme will be assessed through multiple instruments and questionnaires. The instruments are validated and used in community-dwelling older patients with cognitive disorders. Additional data will be collected on the time spent at home (home time); total number of days alive and out of the hospital or a skilled nursing facility in the 6 months following the randomisation at the $\mathrm{ED},{ }^{31}$ the number of transfers (home $\leftrightarrow$ hospital) and the number of healthcare professionals involved.

Cost data will be collected, as described by Drummond et al, including the costs to the healthcare system, patients and families, and other sectors. ${ }^{32}$ The volume of care use will be extracted from hospital files and combined with the reference cost values, as provided by the cost guidance module of the Dutch National Health Care Institute. ${ }^{33}$

\section{Process evaluation}

A process evaluation will be conducted as part of the feasibility study to understand the barriers and facilitators to participate and to gain an understanding of the experiences and perceptions of Hospital at Home care of participants and healthcare professionals. From all eligible patients who declined to participate, data concerning the patient characteristics and reasons for non-participation will be collected. At the end of the trial, a representative sample of participants and/or their informal caregivers will be invited for an interview to evaluate their experiences receiving Hospital at Home care. The interviewer will not be a an active member of the research team or involved in day-to-day care and will explore independently how the participants perceived Hospital at Home care, including the contact with the health professionals and the impact of Hospital at Home care on their lives and their caregivers' personal lives. In case of participant dropout, efforts will be made to obtain an understanding of why the participants did not complete the trial.

In addition, a representative sample of healthcare professionals, consisting of physicians and nurses working in the ED, physicians and nurses providing the Hospital at Home care, and general practitioners, will be asked to participate in a face-to-face interview. The healthcare professionals will be asked about their experiences and opinions about the $\mathrm{H} @ \mathrm{H}$ trial and Hospital at Home care. All interviews will be transcribed verbatim, and a framework analysis will be used as the method of qualitative data analysis. ${ }^{34}$

\section{Data management}

All data will be entered in an electronic trial-specific database, with the participants identified by a unique trial number. Confidentiality of participant information 
Table 2 Overview of the content and description of outcome measures and timing of measurements

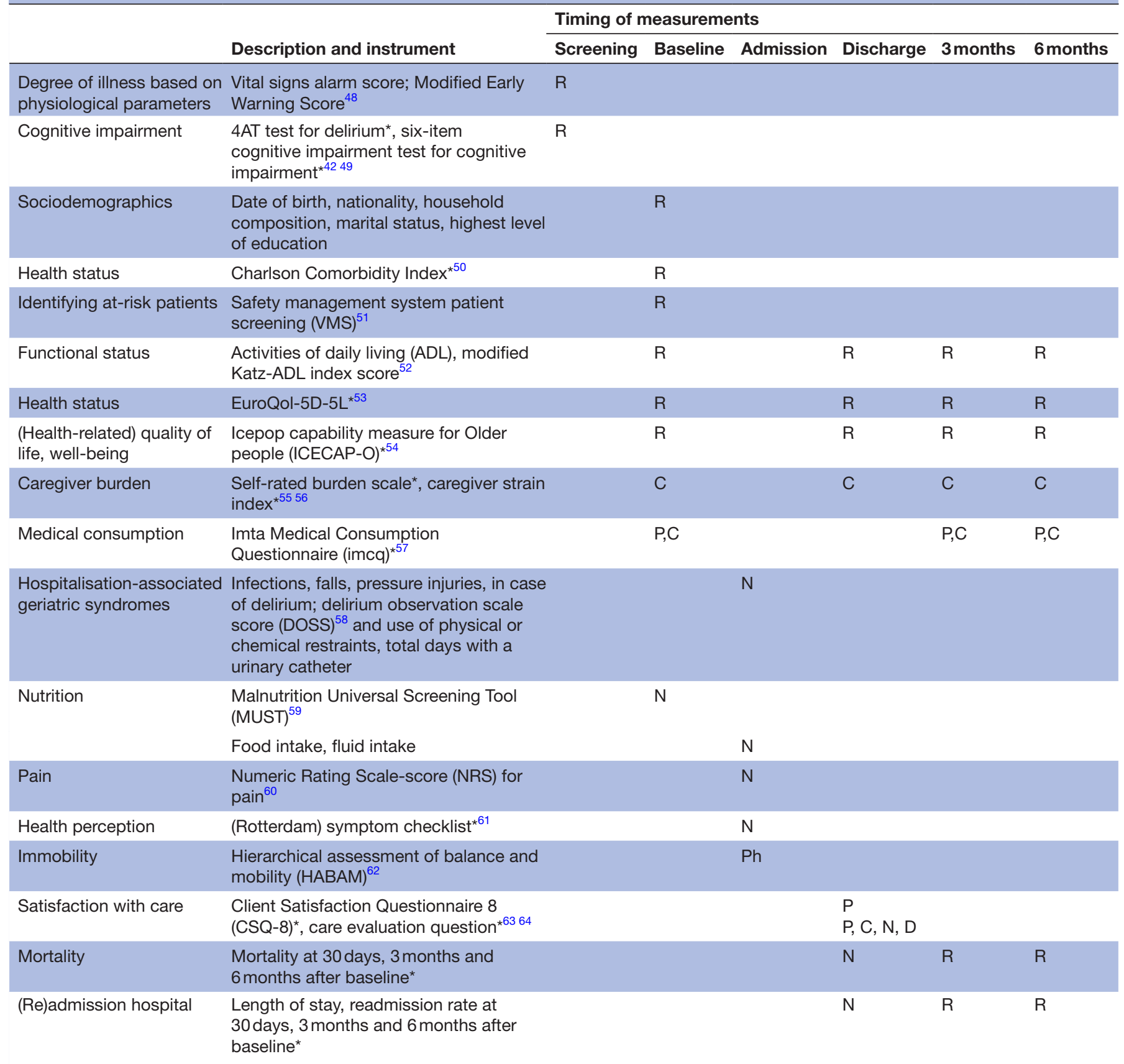

Assessed by: C, caregiver; D, doctor; N, nurse; P, participant; Ph, physiotherapist; R, research nurse.

${ }^{*}$ All assessments are extra for trial purposes and are not part of the medical treatment.

will be maintained throughout the trial. Information can only be traced to the participants by designated researchers. The database will be stored and maintained by Castor Electronic Data Capture, compliant with GCP guidelines and the European Data Protection Directive (Castor Electronic Data Capture, 2017; Ciwit BV, Amsterdam, the Netherlands). Data will be stored for a maximum period of 15 years after the study has ended, according to Dutch law. ${ }^{35}$

\section{Statistical analysis}

The participant flow diagram, according to Consolidated Standards of Reporting Trials (CONSORT) guidelines, ${ }^{36}$ will provide a summary of the recruitment and declination rates in percentage (\%) at baseline, discharge and 3-month and 6-month follow-ups. Distributions of the data at baseline, discharge and 3 and 6 months after randomisation will be explored, with unusual values noted and explained. Variables will be summarised as the n (\%), mean (SD) or median (IQR) for each group to characterise the sample and search for any imbalances. The percentages, means and SD, and medians and IQRs will be calculated to describe the quality of care and the advantages and disadvantages of Hospital at Home care at baseline, discharge and 3-month and 6-month follow-ups. 


\section{Monitoring and participant safety}

Although, the $\mathrm{H} @ \mathrm{H}$ trial is considered to be a low risk trial, the participant safety will be monitored by an independent Data Monitoring Committee (DMC). The DMC will consist of two members: an experienced clinician and an epidemiologist. Members of the DMC are independent of the trial and will discuss each individual participant with serious adverse events. The DMC will receive and review the serious adverse events and evaluate the risk involved with negative outcomes. The DMC is authorised to make recommendations to temporary put on hold or ending the study prematurely when participant safety is an issue, based on their findings. All serious adverse events will be reported to the principal investigator within 24 hours of knowledge of the event and then subsequently reported to the Dutch portal for medical research involving human subjects.

\section{DISCUSSION}

Reducing unwanted hospital admissions in older patients with cognitive impairments and facilitating patient-centred care in a patient's preferred location is a goal worthy of pursuing. This goal aligns with the tenet of the current Dutch government and the advice provided by the Dutch Council for the Environment and Infrastructure: to actively promote and enable people to live independently in their own homes for as long as they desire. ${ }^{37}$ Previous trials and a recent review have confirmed that alternative management strategies for low-risk patients with acute medical conditions conventionally treated through hospitalisation exist with positive impact on patient satisfaction, are effective and can be safely achieved in lower cost settings. $^{22} 2338$

Introducing a Hospital at Home care trajectory in the Netherlands is incited by the principles of value-based healthcare: improving the patients experience of care and as a result of this process reducing the costs. ${ }^{39}$ All countries with an ageing population experience pressure, in terms of shortage of (emergency) hospital beds and rising healthcare costs. Hospital at Home care could be shown beneficial in facilitating higher valued care for patients and their caregivers without additional costs. Benefit should be measured in other outcomes than clinical indicators such as mortality. To illustrate, one of the outcomes of a future RCT could be the time spent at home. Time spent at home has been defined as the total number of days alive and out of the hospital or a skilled nursing facility in the 6 months after hospital admission. ${ }^{31}$ It has been used as a primary outcome in a follow-up study of older patients with acute hospital admissions, and has been demonstrated to be of more importance in older patients. ${ }^{174041}$ Evaluation of time spent a home in this feasibility study could support estimating a sample size based on a patient-relevant outcome in a future RCT.

This study will be the first to investigate the feasibility of providing acute hospital care at home for older patients with cognitive impairment in the Netherlands. Studying
Hospital at Home care and identifying the barriers and facilitators will support the implementation of Hospital at Home care and break new ground for a future RCT investigating the (cost-)effectiveness.

\section{ETHICS AND DISSEMINATION}

The trial will be conducted in accordance with the Declaration of Helsinki 1996, principles of good clinical practice and the University Medical Center of Groningen Research Code. Any protocol amendments will be submitted to the ethics committee. A register of the protocol amendments will be available in the study protocol.

The results of the trial will be reported according to the CONSORT guidelines and will contribute to knowledge of the implementation of Hospital at Home care and patient-centred acute care for older patients with cognitive impairment. The study will also contribute to the knowledge of the transmural cooperation and costs of providing care, in terms of the translocation of hospital care to home. Regularly updates will be published on the study website and in newsletters. Conferences and meetings will be held for all involved healthcare professionals. Participants who requested information on the study will be sent a lay summary. A publication policy will be agreed on with co-applicants. The study findings will be published in relevant peer-reviewed journals.

Acknowledgements The authors thank all care professionals of Alzheimer Nederland, Espria, Icare, TSN, Buurtzorg, umcGroningen thuis, MCZ, Netwerk Dementie Groningen, Netwerk Dementie Drenthe, Gemeente Groningen, WIJ Groningen, Zorgbelang, Healthy Aging Network Northern Netherlands, Stichting Effectieve Ouderenzorg, ZonMW, Deltaplan Dementie, Nationaal Programma Ouderenzorg, Zorg innovatie Forum and the elderly of Denktank 60+ Noord for their time, critical appraisal and advise.

Contributors MAP contributed to the conception and design of the protocol, drafted the manuscript and revised the final manuscript. AHC, BCvM, JCtM and NS made substantial contributions to the conception and design of the protocol. NS contributed to the methodological aspects of the protocol. AHC, BCvM and JCtM contributed to the clinical aspects of the protocol. SEdR conceived the study, developed the protocol together with MAP and wrote the funding applications. All authors critically revised the manuscript and approved the final version of this manuscript.

Funding This study is an independent research funded by ZonMw (project Memorabel, Deltaplan Dementie), project no: 733050401 (contact: memorabel@ zonmw.nl). Additional funding is provided by the Dutch Ministry of Health, Welfare and Sport (Ministerie van Volksgezondheid, Welzijn en Sport) and the University Medical Center Groningen.

Competing interests None declared.

Patient consent Detail has been removed from this case description/these case descriptions to ensure anonymity. The editors and reviewers have seen the detailed information available and are satisfied that the information backs up the case the authors are making.

Ethics approval This study obtained ethical approval from the Medical Research Ethics Committee of the University Medical Center Groningen, April 2017 (ref: 2016.686).

Provenance and peer review Not commissioned; externally peer reviewed.

Open Access This is an Open Access article distributed in accordance with the terms of the Creative Commons Attribution (CC BY 4.0) license, which permits others to distribute, remix, adapt and build upon this work, for commercial use, provided the original work is properly cited. See: http://creativecommons.org/ licenses/by/4.0/ 
(c) Article author(s) (or their employer(s) unless otherwise stated in the text of the article) 2018. All rights reserved. No commercial use is permitted unless otherwise expressly granted.

\section{REFERENCES}

1. Krumholz HM. Post-hospital syndrome - a condition of generalized risk. N Engl J Med 2013;368:100-2.

2. Missildine K, Bergstrom N, Meininger J, et al. Sleep in hospitalized elders: a pilot study. Geriatr Nurs 2010;31:263-71.

3. Brown CJ, Redden DT, Flood KL, et al. The underrecognized epidemic of low mobility during hospitalization of older adults. J Am Geriatr Soc 2009;57:1660-5.

4. Sullivan $\mathrm{DH}$, Sun $\mathrm{S}$, Walls RC. Protein-energy undernutrition among elderly hospitalized patients: a prospective study. JAMA 1999;281:2013-9.

5. Creditor MC. Hazards of hospitalization of the elderly. Ann Intern Med 1993;118:219-23.

6. Inouye SK, Studenski S, Tinetti ME, et al. Geriatric syndromes: clinical, research, and policy implications of a core geriatric concept. J Am Geriatr Soc 2007;55:780-91.

7. Mudge AM, Banks MD, Barnett AG, et al. CHERISH (collaboration for hospitalised elders reducing the impact of stays in hospital): protocol for a multi-site improvement program to reduce geriatric syndromes in older inpatients. BMC Geriatr 2017;17:11.

8. Covinsky KE, Pierluissi E, Johnston CB. Hospitalization-associated disability: "she was probably able to ambulate, but I'm not sure". JAMA 2011;306:1782-93

9. Zisberg A, Shadmi E, Gur-Yaish N, et al. Hospital-associated functional decline: the role of hospitalization processes beyond individual risk factors. J Am Geriatr Soc 2015;63:55-62.

10. Fried LP, Tangen CM, Walston J, et al. Frailty in older adults: evidence for a phenotype. J Gerontol A Biol Sci Med Sci 2001;56:M146-M157.

11. Clegg A, Young J, Iliffe S, et al. Frailty in elderly people. The Lancet 2013;381:752-62.

12. Ferrucci L, Guralnik JM, Studenski S, et al. Designing randomized, controlled trials aimed at preventing or delaying functional decline and disability in frail, older persons: a consensus report. J Am Geriatr Soc 2004;52:625-34.

13. Glover A, Bradshaw LE, Watson N, et al. Diagnoses, problems and healthcare interventions amongst older people with an unscheduled hospital admission who have concurrent mental health problems: a prevalence study. BMC Geriatr 2014;14:1.

14. Pinkert C, Holle B. [People with dementia in acute hospitals. Literature review of prevalence and reasons for hospital admission] Z Gerontol Geriatr 2012;45:728-34.

15. Mukadam N, Sampson EL. A systematic review of the prevalence, associations and outcomes of dementia in older general hospital inpatients. Int Psychogeriatr 2011;23:344-55.

16. Timmons S, Manning E, Barrett A, et al. Dementia in older people admitted to hospital: a regional multi-hospital observational study of prevalence, associations and case recognition. Age Ageing 2015;44:993-9.

17. Bradshaw LE, Goldberg SE, Lewis SA, et al. Six-month outcomes following an emergency hospital admission for older adults with comorbid mental health problems indicate complexity of care needs. Age Ageing 2013;42:582-8.

18. Satizabal CL, Beiser AS, Chouraki V, et al. Incidence of dementia over three decades in the framingham heart study. N Engl J Med 2016;374:523-32.

19. World Health Organization. Dementia fact sheet. $2016 \mathrm{http}: / / \mathrm{www}$. who.int/mediacentre/factsheets/fs362/en/ (accessed 25 Jan 2017).

20. Fried TR, van Doorn C, O'Leary JR, et al. Older person's preferences for home vs hospital care in the treatment of acute illness. Arch Intern Med 2000;160:1501-6.

21. Fried TR, Bradley EH, Towle VR, et al. Understanding the treatment preferences of seriously ill patients. N Engl J Med 2002;346:1061-6.

22. Shepperd S, lliffe S, Doll HA, et al. Admission avoidance hospital at home. Cochrane Database Syst Rev 2016;10.

23. Conley J, O'Brien CW, Leff BA, et al. Alternative strategies to inpatient hospitalization for acute medical conditions: a systematic review. JAMA Intern Med 2016;176:1693-702.

24. Caplan G. Does 'Hospital in the Home' treatment prevent delirium? Aging health 2008;4:69-74.

25. Tibaldi $\mathrm{V}$, Aimonino $\mathrm{N}$, Ponzetto $\mathrm{M}$, et al. A randomized controlled trial of a home hospital intervention for frail elderly demented patients: behavioral disturbances and caregiver's stress. Arch Gerontol Geriatr Suppl 2004;38:431-6.
26. Shepperd S, Cradduck-Bamford A, Butler C, et al. A multi-centre randomised trial to compare the effectiveness of geriatrician-led admission avoidance hospital at home versus inpatient admission. Trials 2017;18:491.

27. Chan AW, Tetzlaff JM, Altman DG, et al. SPIRIT 2013 statement: defining standard protocol items for clinical trials. Ann Intern Med 2013;158:200-7.

28. Aimonino Ricauda N, Tibaldi V, Leff B, et al. Substitutive "hospital at home" versus inpatient care for elderly patients with exacerbations of chronic obstructive pulmonary disease: a prospective randomized, controlled trial. J Am Geriatr Soc 2008;56:493-500.

29. Tibaldi V, Isaia G, Scarafiotti C, et al. Hospital at home for elderly patients with acute decompensation of chronic heart failure: a prospective randomized controlled trial. Arch Intern Med 2009;169:1569-75

30. Worth A, Tierney AJ. Conducting research interviews with elderly people by telephone. J Adv Nurs 1993;18:1077-84.

31. Quinn TJ, Dawson J, Lees JS, et al. Time spent at home poststroke: "home-time" a meaningful and robust outcome measure for stroke trials. Stroke 2008;39:231-3.

32. Drummond MF, Sculpher MJ, Claxton K, et al. Methods for the economic evaluation of health care programmes. Oxford university press, 2015.

33. Hakkaart-van Roijen L, Van der Linden N, Bouwmans C, et al. Kostenhandleiding. Methodologie van kostenonderzoek en referentieprijzen voor economische evaluaties in de gezondheidszorg. Institute for Medical Technology Assessment, 2015.

34. Ritchie J, Spencer L. Qualitative data analysis for applied policy research. The Qualitative Researcher's Companion 2002;573:305-29.

35. Overheid.nl. Wet bescherming persoonsgegevens. $2000 \mathrm{http}: / /$ wetten.overheid.nl/BWBR0011468/2017-07-01/0/ (accessed 25 Oct 2017)

36. Schulz KF, Altman DG, Moher D. CONSORT 2010 statement: updated guidelines for reporting parallel group randomised trials. Trials 2010;11:32.

37. Raad voor de leefomgeving en infrastructuur. Langer zelfstandig een gedeelde opgave van wonen, zorg en welzijn. $2014 \mathrm{http}: / /$ www.rli.nl/sites/default/files/rli-briefadvieslangerzelfstandigeengede eldeopgavevanwonenzorgenwelzijndef23-113-30_0.pdf (accessed 25 Oct 2017)

38. Caplan GA, Sulaiman NS, Mangin DA, et al. A meta-analysis of "hospital in the home". Med J Aust 2012;197:512-9.

39. Porter ME. What is value in health care? N Engl J Med 2010;363:2477-81.

40. Sayer C. "Time spent at home" - a patient-defined outcome. NEJM Catalyst 2016.

41. Groff AC, Colla CH, Lee TH. Days spent at home - a patient-centered goal and outcome. N Engl J Med 2016;375:1610-2.

42. Bellelli G, Morandi A, Davis DH, et al. Validation of the 4AT, a new instrument for rapid delirium screening: a study in 234 hospitalised older people. Age Ageing 2014;43:496-502.

43. Katzman R, Brown T, Fuld P, et al. Validation of a short orientationmemory-concentration test of cognitive impairment. Am J Psychiatry 1983;140:734-9.

44. Shepperd S, Harwood D, Jenkinson C, et al. Randomised controlled trial comparing hospital at home care with inpatient hospital care. I: three month follow up of health outcomes. BMJ 1998;316:1786-91.

45. Cei M, Bartolomei C, Mumoli N. In-hospital mortality and morbidity of elderly medical patients can be predicted at admission by the Modified Early Warning Score: a prospective study. Int J Clin Pract 2009;63:591-5.

46. Dundar ZD, Ergin M, Karamercan MA, et al. Modified Early Warning Score and vitalpac early warning score in geriatric patients admitted to emergency department. Eur J Emerg Med 2016;23:406-412.

47. Ridley $S$. The recognition and early management of critical illness. Ann R Coll Surg Engl 2005;87:315-22.

48. Subbe CP, Kruger M, Rutherford P, et al. Validation of a Modified Early Warning Score in medical admissions. QJM 2001;94:521-6.

49. et alAmerican College of Emergency Physicians, American Geriatrics Society, Emergency Nurses Association, Geriatric emergency department guidelines. Ann Emerg Med 2014;63:e7-25

50. Charlson ME, Pompei P, Ales KL, et al. A new method of classifying prognostic comorbidity in longitudinal studies: development and validation. J Chronic Dis 1987;40:373-83.

51. Oud F, de Rooij S, Schuurman T, et al. [Predictive value of the VMS theme 'Frail elderly': delirium, falling and mortality in elderly hospital patients]. Ned Tijdschr Geneeskd 2014:159:A8491.

52. Katz S, Ford AB, Moskowitz RW, et al. Studies of illness in the aged: the index of $A D L$ : a standardized measure of biological and psychosocial function. JAMA 1963;185:914-9. 
53. Herdman M, Gudex C, Lloyd A, et al. Development and preliminary testing of the new five-level version of EQ-5D (EQ-5D-5L). Qual Life Res 2011;20:1727-36.

54. Makai P, Brouwer WB, Koopmanschap MA, et al. Capabilities and quality of life in Dutch psycho-geriatric nursing homes: an exploratory study using a proxy version of the ICECAP-O. Qual Life Res 2012;21:801-12.

55. van Exel NJ, Scholte op Reimer WJ, Brouwer WB, et al. Instruments for assessing the burden of informal caregiving for stroke patients in clinical practice: a comparison of CSI, CRA, SCQ and self-rated burden. Clin Rehabil 2004;18:203-14.

56. Robinson BC. Validation of a caregiver strain index. $J$ Gerontol 1983:38:344-8

57. Bouwmans C, Hakkaart-van Roijen L, Koopmanschap M, et al. Handleiding iMTA Medical Cost Questionnaire (iMCQ). Rotterdam: iMTA, Erasmus Universiteit Rotterdam, 2013.

58. Gavinski K, Carnahan R, Weckmann M. Validation of the delirium observation screening scale in a hospitalized older population. $J$ Hosp Med 2016;11:494-7.
59. Stratton RJ, Hackston A, Longmore D, et al. Malnutrition in hospita outpatients and inpatients: prevalence, concurrent validity and ease of use of the 'malnutrition universal screening tool' ('MUST') for adults. Br J Nutr 2004;92:799-808.

60. Hawker GA, Mian S, Kendzerska T, et al. Measures of adult pain: Visual Analog Scale for Pain (VAS Pain), Numeric Rating Scale for Pain (NRS Pain), McGill Pain Questionnaire (MPQ), Short-Form McGill Pain Questionnaire (SF-MPQ), Chronic Pain Grade Scale (CPGS), Short Form-36 Bodily Pain Scale (SF-36 BPS), and Measure of Intermittent and Constant Osteoarthritis Pain (ICOAP). Arthritis Care Res 2011;63(S11):S240-2.

61. de Haes JC, van Knippenberg FC, Neijt JP. Measuring psychological and physical distress in cancer patients: structure and application of the rotterdam symptom checklist. Br J Cancer 1990;62:1034-8.

62. MacKnight C, Rockwood K. A hierarchical assessment of balance and mobility. Age Ageing 1995;24:126-30.

63. Attkisson CC, Greenfield TK. The UCSF Client Satisfaction Scales: I. The Client Satisfaction Questionnaire-8, 1999.

64. Reichheld FF. The one number you need to grow. Harv Bus Rev 2003;81:46-55. 